\title{
The Thomas-Fermi-von Weizsäcker Theory of Atoms and Molecules ${ }^{\star}$
}

\author{
Rafael Benguria ${ }^{1}$, Haim Brezis ${ }^{2}$, and Elliott H. Lieb ${ }^{3}$
}

1. The Rockefeller University, New York, NY 10021, USA, on leave from Universidad de Chile, Santiago, Chile

2. Département de Mathématiques, Université Paris VI, F-75230, Paris Cedex 05, France

3. Departments of Mathematics and Physics, Princeton University, Princeton, NJ 08544, USA

\begin{abstract}
We place the Thomas-Fermi-von Weizsäcker model of atoms on a firm mathematical footing. We prove existence and uniqueness of solutions of the Thomas-Fermi-von Weizsäcker equation as well as the fact that they minimize the Thomas-Fermi-von Weizsäcker energy functional. Moreover, we prove the existence of binding for two very dissimilar atoms in the frame of this model.
\end{abstract}

\section{Introduction}

The Thomas-Fermi theory of atoms [1] (TF), attractive because of its simplicity, is not satisfactory because it yields an electron density with incorrect behavior very close and very far from the nucleus. Moreover, it does not allow for the existence of molecules. In order to correct this, von Weizsäcker [2] suggested the addition of an inhomogeneity correction

$$
U_{w}(\rho)=C_{w}(\nabla \rho)^{2} / \rho
$$

to the kinetic energy density. Here $c_{w}=h^{2} /\left(32 \pi^{2} m\right)$, where $m$ is the mass of the electron. This correction has also been obtained as the first order correction to the TF kinetic energy in a semi-classical approximation to the Hartree-Fock theory [3].

The Thomas-Fermi-von Weizsäcker (henceforth TFW) energy functional for nuclei of charges $z_{i}>0$ (which need not be integral) located at $R_{i}, i=1, \ldots, k$ is defined by

$$
\begin{aligned}
\xi(\rho)= & \left(3 \pi^{2}\right)^{-2 / 3} \int\left(\nabla \rho^{1 / 2}(x)\right)^{2} d x+\frac{3}{5} \int \rho(x)^{5 / 3} d x \\
& -\int V(x) \rho(x) d x+\frac{1}{2} \iint \rho(x) \rho(y)|x-y|^{-1} d x d y,
\end{aligned}
$$

in units in which $h^{2}(8 m)^{-1}(3 / \pi)^{2 / 3}=1$ and $|e|=1$. Here $\rho(x) \geqq 0$ is the electron

* Research supported by U. S. National Science Foundation under Grants MCS78-20455 (R. B.), PHY-7825390 A 01 (H. B. and E. L.), and Army Research Grant DAH 29-78-6-0127 (H. B.) 
density, and

$$
V(x)=\sum_{i=1}^{k} z_{i}\left|x-R_{i}\right|^{-1} .
$$

While the pure TF problem has been placed on a rigorous mathematical footing [1], no parallel study has been made for the TFW problem. Such a study was undertaken in the Ph.D. thesis of one of us [4]; in this paper some of the results of [4] will be presented together with some newer results.

In this article we will study a rather more general functional, which contains the TFW energy functional (2) as a particular case. In fact, for $\rho(x) \geqq 0$ and $V(x)$ given by (3), let us introduce the functional

$$
\xi_{p}(\rho)=\int\left|\nabla \rho^{1 / 2}(x)\right|^{2} d x+\frac{1}{p} \int \rho^{p}(x) d x-\int V(x) \rho(x) d x+D(\rho, \rho),
$$

where

$$
D(f, g) \equiv \frac{1}{2} \iint f(x) g(y)|x-y|^{-1} d x d y,
$$

for $1<p<\infty$.

We shall be concerned with the following problem

$$
\operatorname{Min}\left\{\xi_{p}(\rho) \mid \rho \in L^{1} \cap L^{p}, \rho(x) \geqq 0, \nabla \rho^{1 / 2} \in L^{2} \text { and } \int \rho(x) d x=\lambda\right\},
$$

where $\lambda$ is a given positive constant, which, physically, is the total electron number.

Our main result is the following:

Theorem 1. There is a critical value $0<\lambda_{c}<\infty$ depending only on $p$ and $V$ such that

(a) If $\lambda \leqq \lambda_{c}$, Problem (I) has a unique solution.

(b) If $\lambda>\lambda_{c}$, Problem (I) has no solution. In addition,

(c) When $p \geqq \frac{4}{3}$, then $\lambda_{c} \geqq Z \equiv \sum_{i=1}^{k} z_{i}$.

(d) When $p \geqq \frac{5}{3}$ and $k=1$ (atomic case), then $\lambda_{c}>Z$.

Remark 1. Partial results were previously obtained by one of us. Namely in [4] it is proved that for the atomic case $(k=1)$ and $p=\frac{5}{3}$, Problem (I) has a solution if $\lambda \leqq Z$.

Remark 2. Some of the open problems which are raised by our developments are the following:

(i) Suppose $k \geqq 2$ (molecular case) and $p=\frac{5}{3}$. Is $\lambda_{c}>Z$ ?

(ii) Find estimates for $\lambda_{c}$.

(iii) Is there binding for atoms?

With respect to the third problem, there is a non-rigorous argument of Balàzs [5] that indicates the possibility of binding for homopolar diatomic molecule in the TFW theory. Also, Gombás [6] applied TFW (including exchange corrections) to study the $N_{2}$-molecule (i.e., $z_{1}=z_{2}=7$ ) and found numerically that 
there is binding. He actually computed the distance between the two centers to be $1.39 \AA$ for the configuration of minimum energy. We do not give a proof of binding in the homopolar case, but we will prove that binding occurs for two very dissimilar atoms.

Remark 3. Theorem 1 obviously holds if we replace $\xi_{p}$ by

$$
\xi_{p}(\rho)=c_{1} \int\left|\nabla \rho^{1 / 2}(x)\right|^{2} d x+c_{2} \int \rho^{p}(x) d x-\int V(x) \rho(x) d x+D(\rho, \rho),
$$

where $c_{1}$ and $c_{2}$ are positive constants.

The proof of Theorem 1 is divided into several steps. In Sect. 1 we describe some basic properties of $\xi_{p}(\rho)$. In Sect. 2 we consider the problem

$$
\operatorname{Min}\left\{\xi_{p}(\rho) \mid \rho \in D_{p}\right\}
$$

where $D_{p}=\left\{\rho \mid \rho(x) \geqq 0, \rho \in L^{3} \cap L^{p}, \nabla \rho^{1 / 2} \in L^{2}, D(\rho, \rho)<\infty\right\}$, and we prove that the minimum is achieved at a unique $\rho_{0}$. Note that $D_{p}$ contains $\{\rho \mid \rho \geqq 0$, $\left.\rho \in L^{3} \cap L^{p} \cap L^{1}, \nabla \rho^{1 / 2} \in L^{2}\right\}$. We derive the Euler equation for $\rho_{0}$. More precisely we set $\psi=\rho_{0}^{1 / 2}$ and we show that

$$
-\Delta \psi+\psi^{2 p-1}=\varphi \psi
$$

where

$$
\varphi(x)=V(x)-\int \psi^{2}(y)|x-y|^{-1} d y .
$$

In Sect. 3 we prove that $\psi \in L^{2}$ and we obtain some further properties of $\psi(\psi$ is continuous, $\psi(x) \rightarrow 0$ as $|x| \rightarrow \infty$, etc.). In Sect. 4 we show that if $p \geqq \frac{4}{3}$ then $\int \psi^{2}(x) d x \geqq Z$. In Sect. 5 we show that if $p \geqq \frac{5}{3}$ and $k=1$ then $\int \psi^{2}(x) d x>Z$. In addition $\psi(x) \leqq M e^{-\delta|x|^{1 / 2}}$ for some appropriate constants $M$ and $\delta>0$. In Sect. 6 we prove that for every $\lambda$

$$
E(\lambda) \equiv \operatorname{Inf}\left\{\xi_{p}(\rho) \mid \int \rho(x) d x=\lambda\right\}=\operatorname{Inf}\left\{\xi_{p}(\rho) \mid \int \rho(x) d x \leqq \lambda\right\}
$$

and we conclude the proof of Theorem 1 . We also show that $E(\lambda)$ is convex, monotone non-increasing and that $E(\lambda)$ has a finite slope at $\lambda=0$. This slope is the ground state energy of the corresponding one electron Schrödinger Equation. Using this last fact, binding for dissimilar atoms is proved.

\section{Some Basic Properties of $\xi_{p}$}

In Lemma 2, 3, 4 some properties which are useful in the study of Problem (I) are summarized.

Lemma 2. For every $\varepsilon>0$, there is a constant $C_{\varepsilon}$, depending on $V$ but independent of $\rho$, such that

$$
\int V(x) \rho(x) d x \leqq \varepsilon\|\rho\|_{3}+C_{\varepsilon} D(\rho, \rho)^{1 / 2},
$$

for every $\rho \geqq 0$.

Proof. Let $\delta>0$ be a small constant and let $\zeta(x)$ be a smooth function such that 
$0 \leqq \zeta \leqq 1$ and

$$
\zeta(x)= \begin{cases}1 & \text { on } \bigcup_{i=1}^{k} B_{\delta}\left(R_{i}\right) \\ 0 & \text { outside } \bigcup_{i=1}^{k} B_{2 \delta}\left(R_{i}\right),\end{cases}
$$

where $B_{\delta}\left(R_{i}\right)$ is the ball of radius $\delta$ and centered at $R_{i} . \delta$ is chosen such that all these ball $B_{2 \delta}$ are disjoint. Let $V=V \zeta+V(1-\zeta) \equiv V_{1}+V_{2}$. Clearly $V_{1} \in L^{3 / 2}$ and by choosing $\delta$ small enough we may assume that $\left\|V_{1}\right\|_{3 / 2}<\varepsilon$. Thus

$$
\int V_{1}(x) \rho(x) d x \leqq \varepsilon\|\rho\|_{3} .
$$

On the other hand define the operator $B$ to be

$$
(B \rho)(x)=\int \rho(y)|x-y|^{-1} d y,
$$

so that (in the sense of distributions) we have

$$
-\Delta(B \rho)=4 \pi \rho \text {. }
$$

Thus,

$$
\int|\nabla(B \rho)|^{2} d x=8 \pi D(\rho, \rho)
$$

We deduce from (11) and Sobolev's inequality that

$$
\|B \rho\|_{6} \leqq C D(\rho, \rho)^{1 / 2} \text {. }
$$

Consequently,

$$
\begin{aligned}
\int V_{2}(x) \rho(x) d x & =\frac{1}{4 \pi} \int\left(-\Delta V_{2}\right)(x)(B \rho)(x) d x \\
& \leqq C\left\|\Delta V_{2}\right\|_{6 / 5} D(\rho, \rho)^{1 / 2}
\end{aligned}
$$

(note that $\Delta V_{2} \in C_{0}^{\infty}$ ). Combining (9) and (13) we obtain the conclusion.

Lemma 3. There exist positive constants $\alpha$ and $C$ such that

$$
\xi_{p}(\rho) \geqq \alpha\left(\|\rho\|_{3}+\|\rho\|_{p}^{p}+\left\|\nabla \rho^{1 / 2}\right\|_{2}^{2}+D(\rho, \rho)\right)-C
$$

Proof. Use Lemma 2 and Sobolev's inequality.

Lemma 4. $\xi_{p}(\rho)$ is strictly convex.

Proof. The only non-standard fact is that the function $\rho \rightarrow \int\left|\nabla \rho^{1 / 2}\right|^{2} d x$ is convex (or equivalently subadditive). Indeed let $\rho_{1}, \rho_{2} \in D_{p}$ and set $\psi_{1}=\rho^{1 / 2}$, $\psi_{2}=\rho_{2}^{1 / 2}, \psi_{3}=\left(\alpha \rho_{1}+(1-\alpha) \rho_{2}\right)^{1 / 2}$ with $0<\alpha<1$. Thus,

$$
\begin{aligned}
\psi_{3} \nabla \psi_{3} & =\alpha \psi_{1} \nabla \psi_{1}+(1-\alpha) \psi_{2} \nabla \psi_{2} \\
& =\left(\alpha^{1 / 2} \psi_{1}\right)\left(\alpha^{1 / 2} \nabla \psi_{1}\right)+\left[(1-\alpha)^{1 / 2} \psi_{2}\right]\left[(1-\alpha)^{1 / 2} \nabla \psi_{2}\right]
\end{aligned}
$$

and by Cauchy-Schwarz inequality

$$
\psi_{3} \nabla \psi_{3} \leqq\left(\alpha \psi_{1}^{2}+(1-\alpha) \psi_{2}^{2}\right)^{1 / 2}\left(\alpha\left|\nabla \psi_{1}\right|^{2}+(1-\alpha)\left|\nabla \psi_{2}\right|^{2}\right)^{1 / 2}
$$


and therefore,

$$
\left|\nabla \psi_{3}\right|^{2} \leqq \alpha\left|\nabla \psi_{1}\right|^{2}+(1-\alpha)\left|\nabla \psi_{2}\right|^{2}
$$

\section{Minimization of $\xi_{p}(\rho)$ with $\rho \in D_{p}-$ The Euler Equation}

We start with

Lemma 5. $\operatorname{Min}\left\{\xi_{p}(\rho) \mid \rho \in D_{p}\right\}$ is achieved at some $\rho_{0} \in D_{p}$.

Proof. Let $\rho_{n} \in D_{p}$ be a minimizing sequence. By Lemma 3 we have

$$
\left\|\rho_{n}\right\|_{3} \leqq C,\left\|\rho_{n}\right\|_{p} \leqq C,\left\|\nabla \rho_{n}^{1 / 2}\right\|_{2} \leqq C, D\left(\rho_{n}, \rho_{n}\right) \leqq C .
$$

Therefore, we may extract a subsequence, still denoted by $\rho_{n}$, such that

$$
\begin{gathered}
\rho_{n} \rightarrow \rho_{0} \text { weakly in } L^{3} \text { and in } L^{p}, \\
\quad \rho_{n} \rightarrow \rho_{0} \text { a.e., } \\
\nabla \rho_{n}^{1 / 2} \rightarrow \nabla \rho_{0}^{1 / 2} \text { weakly in } L^{2} .
\end{gathered}
$$

((15) relies on the fact that if $\Omega$ is a bounded smooth domain then $H^{1}(\Omega)$ is relatively compact in $L^{2}(\Omega)$. (14) and (16) implies that $\left\{\rho_{n}^{1 / 2}\right\}$ is bounded in $H^{1}(\Omega)$. Hence $\left\{\rho_{n}^{1 / 2}\right\}$ has a subsequence converging in $L^{2}(\Omega)$ and a.e.). Hence,

$$
\begin{aligned}
& \lim \inf \int\left|\nabla \rho_{n}^{1 / 2}\right|^{2} d x \geqq \int\left|\nabla \rho_{0}^{1 / 2}\right|^{2} d x, \\
& \lim \inf \int \rho_{n}^{p}(x) d x \geqq \int \rho_{0}^{p}(x) d x,
\end{aligned}
$$

$\lim \inf D\left(\rho_{n}, \rho_{n}\right) \geqq D\left(\rho_{0}, \rho_{0}\right)$ (by Fatou's Lemma).

We now prove that

$$
\int V(x) \rho_{n}(x) d x \rightarrow \int V(x) \rho_{0}(x) d x
$$

As in the proof of Lemma 2, we write $V=V_{1}+V_{2}$. Clearly,

$$
\int V_{1}(x) \rho_{n}(x) d x \rightarrow \int V_{1}(x) \rho_{0}(x) d x,
$$

since $V_{1} \in L^{3 / 2}$. On the other hand

$$
\int V_{2}(x) \rho_{n}(x) d x=-\frac{1}{4 \pi} \int\left(\Delta V_{2}\right)\left(B \rho_{n}\right) d x
$$

It follows from (12) that $B \rho_{n} \rightarrow B \rho_{0}$ weakly in $L^{6}$. Thus,

$$
\int V_{2}(x) \rho_{n}(x) d x \rightarrow \int V_{2}(x) \rho_{0}(x) d x .
$$

Hence,

$$
\xi_{p}\left(\rho_{0}\right) \leqq \lim \inf \xi_{p}\left(\rho_{n}\right)=\operatorname{Inf}\left\{\xi_{p}(\rho) \mid \rho \in D_{p}\right\} .
$$

We now derive the Euler Equation satisfied by $\rho_{0}$. Set $\psi=\rho_{0}^{1 / 2}$.

Lemma 6. The minimizing $\rho_{0}$ satisfies

$$
-\Delta \psi+\psi^{2 p-1}=\varphi \psi
$$


where

$$
\varphi=V-B \psi^{2}
$$

and (17) holds in the sense of distributions.

Proof. So far, we know that $\psi \in L^{6} \cap L^{2 p}, \nabla \psi \in L^{2}$ and $B \psi^{2} \in L^{6}$. Since $V \in L_{\text {loc }}^{2}$, it follows that $\varphi \in L_{\text {loc }}^{2}$ and thus $\varphi \psi \in L_{\mathrm{loc}}^{1}$. On the other hand $\psi^{2 p-1} \in L_{\mathrm{loc}}^{1}$ (since $\left.\psi \in L^{2 p}\right)$. Therefore, (17) has a meaning in the sense of distributions. Consider the set $\tilde{D}=\left\{\zeta \in L^{6} \cap L^{2 p} \mid \nabla \zeta \in L^{2}\right.$ and $\left.D(\zeta, \zeta)<\infty\right\}$. (Note that we do not assume $\zeta \geqq 0$.) If $\zeta \in \tilde{D}$, then $\rho=\zeta^{2} \in D_{p}$ and

$$
\xi_{p}(\rho)=\int|\nabla \zeta|^{2} d x+\frac{1}{p} \int \zeta^{2 p} d x-\int V \zeta^{2}+D(\zeta, \zeta) \equiv \phi(\zeta)
$$

Indeed it suffices to recall that $\nabla \rho^{1 / 2}=\nabla|\zeta|=\nabla \zeta(\operatorname{sgn} \zeta)$ (see [7]). Therefore, we find for every $\zeta \in \tilde{D}$

$$
\phi(\psi) \leqq \phi(\zeta)
$$

Let $\eta \in C_{0}^{\infty}$; using the fact that $d /\left.d t \phi(\psi+t \eta)\right|_{t=0}=0$ we conclude easily that

$$
\int \nabla \psi \cdot \nabla \eta d x+\int \psi^{2 p-1} \eta d x=\int \varphi \psi \eta d x .
$$

\section{Proof that the Minimizing $\psi \in L^{2}$ and Further Properties of $\psi$}

We first prove that the minimizing $\psi$ is continuous:

Lemma 7. $\psi$ is continuous on $\mathbb{R}^{3}$; more precisely $\psi \in C^{0, \alpha}$ for every $\alpha<1$ (i.e., for every bounded set $\Omega \subset \mathbb{R}^{3}$, there is a constant $M$ such that $|\psi(x)-\psi(y)| \leqq M|x-y|^{\alpha}$ $\forall x, y \in \Omega)$.

Proof. We already know that $B \psi^{2} \in L^{6}$ and (clearly) $V \in L_{\text {loc }}^{3-\delta}(\forall \delta>0)$. Consequently, $\varphi \in L_{\text {loc }}^{3-\delta}(\forall \delta>0)$. Since $\psi \in L^{6}$, it follows that $\varphi \psi \in L_{\text {loc }}^{2-\delta}(\forall \delta>0)$. Therefore, we have

$$
-\Delta \psi \leqq f
$$

where $f=\varphi \psi \in L_{\text {loc }}^{2-\delta}(\forall \delta>0)$ and in particular $f \in L_{\text {loc }}^{q}$ for some $q>3 / 2$. We may, therefore, apply a result of Stampacchia (see [7], Théorème 5.2) to conclude that $\psi \in L_{\mathrm{loc}}^{\infty}$. Going back to (17) and using the fact that $\psi \in L_{\mathrm{loc}}^{\infty}$, we now see that $\Delta \psi \in L_{\text {loc }}^{3-\delta}(\forall \delta>0)$. The standard elliptic regularity theory [8] implies that $\psi \in C^{0, \alpha}$ for every $\alpha<1$.

We now prove an important property of $\psi$, namely, $\psi \in L^{2}$. Note that such a fact cannot be deduced from the knowledge that $\rho_{0} \in D_{p}$. It is easy to construct a function $\rho \geqq 0$ such that $\rho \in L^{3} \cap L^{p}, \nabla \rho^{1 / 2} \in L^{2}, D(\rho, \rho)<\infty$ and $\int \rho(x) d x=\infty$.

Lemma 8. $\psi \in L^{2}$.

Proof. Suppose, by contradiction, that $\int \psi^{2}(x) d x=\infty$. Choose $r_{1}>\underset{1 \leqq i \leqq k}{\operatorname{Max}}\left|R_{i}\right|$ such that

$$
\int_{|x|<r_{1}} \psi^{2}(x) d x \geqq Z+2 \delta
$$


for some $\delta>0$. We, thus, have

$$
\begin{aligned}
\left(B \psi^{2}\right)(x)= & \int \psi^{2}(y)|x-y|^{-1} d y \geqq \int_{|y|<r_{1}} \psi^{2}(y)(|x|+|y|)^{-1} d y \\
& \geqq(Z+2 \delta) /\left(|x|+r_{1}\right) .
\end{aligned}
$$

Therefore,

$$
\varphi(x)=V(x)-\left(B \psi^{2}\right)(x) \leqq \frac{Z}{|x|-r_{1}}-\frac{Z+2 \delta}{|x|+r_{1}},
$$

for $|x|>r_{1}$. Consequently, there is some $r_{2}>r_{1}$ such that

$$
\varphi(x) \leqq-\delta|x|^{-1},
$$

for $|x|>r_{2}$. It follows from (17) that

$$
-\Delta \psi+\delta|x|^{-1} \psi \leqq 0,
$$

for $|x|>r_{2}$. We now use a comparison argument. Set

$$
\tilde{\psi}(x)=M e^{-2(\delta|x|)^{1 / 2}},
$$

where $M>0$ is a constant. An easy computation shows that

$$
-\Delta \tilde{\psi}+\delta|x|^{-1} \tilde{\psi} \geqq 0,
$$

for $x \neq 0$. Hence, by (19) and (20) we have

$$
-\Delta(\psi-\tilde{\psi})+\delta|x|^{-1}(\psi-\tilde{\psi}) \leqq 0,
$$

for $|x|>r_{2}$. We fix $M$ in such a way that

$$
\psi(x) \leqq \tilde{\psi}(x),
$$

for $|x|=r_{2}$ (this is possible since $\psi \in L_{\text {loc }}^{\infty}$ ). It follows from (21), (22) and the maximum principle that

$$
\psi(x) \leqq \tilde{\psi}(x),
$$

for $|x|>r_{2}$. Since we only know that $\psi(x) \rightarrow 0$ as $|x| \rightarrow \infty$ in a weak sense (namely $\psi \in L^{6}$ ), we must justify (23). We use a variant of Stampacchia's method. Fix $\zeta(x) \in C_{0}^{\infty}$ with $0 \leqq \zeta \leqq 1$ and

$$
\zeta(x)= \begin{cases}1 & \text { for }|x|<1 \\ 0 & \text { for }|x|>2\end{cases}
$$

Set $\zeta_{n}(x)=\zeta(x / n)$. Multiplying (21) by $\zeta_{n}(\psi-\tilde{\psi})^{+}$(here we set $t^{+}=\operatorname{Max}(t, 0)$ ) and integrating on $\left[|x|>r_{2}\right]$, we find

$$
\int_{|x|>r_{2}} \nabla(\psi-\tilde{\psi})\left[\nabla \zeta_{n}(\psi-\tilde{\psi})^{+}+\zeta_{n} \nabla(\psi-\tilde{\psi})^{+}\right] d x+\int_{|x|>r_{2} \mid} \frac{\delta}{|x|}\left|(\psi-\tilde{\psi})^{+}\right|^{2} \zeta_{n} d x \leqq 0 .
$$

In particular it follows that

$$
\int_{|x|>r_{2}} \frac{\delta}{|x|}\left|(\psi-\tilde{\psi})^{+}\right| \zeta^{2} \zeta_{n} d x \leqq \frac{1}{2} \int_{|x|>r_{2}}\left|(\psi-\tilde{\psi})^{+}\right|^{2} \Delta \zeta_{n} d x
$$


But we have

$$
\begin{aligned}
\int_{|x|>\boldsymbol{r}_{2}}\left|(\psi-\tilde{\psi})^{+}\right| \Delta \zeta_{n} d x & \leqq \frac{C}{n^{2}} \int_{n<|x|<2 n} \psi^{2}(x) d x \\
& \leqq C\left[\int_{n<|x|<2 n} \psi^{6}(x) d x\right]^{1 / 3}
\end{aligned}
$$

by Hölder's inequality. Since $\psi \in L^{6}$, we conclude that the right side in (24) tends to zero as $n \rightarrow \infty$. Consequently,

$$
\int_{|x|>r_{2}} \delta|x|^{-1}\left|(\psi-\tilde{\psi})^{+}\right|^{2} d x=0
$$

and so $\psi \leqq \tilde{\psi}$ for $|x|>r_{2}$. In particular $\int \psi^{2}(x) d x<\infty$, a contradiction with the initial assumption.

We now indicate some further properties of $\psi$.

Lemma 9. $\psi$ is bounded on $\mathbb{R}^{3}, \psi(x) \rightarrow 0$ as $|x| \rightarrow \infty$ and $\psi \in H^{2}$.

Proof. By (17) we have,

$$
-\Delta \psi \leqq V \psi
$$

and so

$$
-\Delta \psi+\psi \leqq(V+1) \psi
$$

Clearly, $(V+1) \psi \in L^{2}$ and so

$$
\psi \leqq(-\Delta+I)^{-1}[(V+1) \psi]
$$

As is well known, the right side in (26) is bound and tends to zero as $|x| \rightarrow \infty$. Finally, note that $\psi^{2 p-1} \leqq C \psi$ for some constant $C$ and $\left(B \psi^{2}\right) \psi \in L^{2}$ (since $\psi \in L^{3}$ and $\left.B \psi^{2} \in L^{6}\right)$. Therefore, we conclude that $\Delta \psi \in L^{2}$ and so $\psi \in H^{2}$.

Lemma 10. $\psi>0$ everywhere and $\psi$ is $C^{\infty}$ except at $x=R_{i}(1 \leqq i \leqq k)$.

Proof. From (17) we have,

$$
-\Delta \psi+a \psi=0
$$

where $a \in L_{\text {loc }}^{q}$ and $q>3 / 2$. It follows from Harnack's inequality (see e.g. [9] Corollary 5.3) that either $\psi>0$ everywhere or $\psi \equiv 0$. We now prove that $\psi \neq \equiv$ by checking that $\operatorname{Min}\left\{\xi_{p}(\rho) \mid \rho \in D_{p}\right\}<0$. It clearly suffices to consider the case where $V(x)=z_{1}|x|^{-1}$. Take the trial function $\rho^{1 / 2}(x)=\gamma \exp \left[-z_{1}|x| / 4\right]$. The terms in $\rho$ which are homogeneous of degree one are $-\gamma^{2} z_{1}^{2} / 4$. The remaining terms are proportional to $\gamma^{s}, s>2$. Hence for $\gamma$ sufficiently small, $\xi_{p}(\rho)<0$. Finally, the fact that $\psi$ is $C^{\infty}$ (except at $R_{i}$ ) follows easily from (17) by a standard bootstrap argument.

Remark. When $p \geqq 3 / 2$, there is a simple estimate for $\psi$, namely

$$
\psi^{2(p-1)}(x) \leqq V(x)
$$


for every $x$. Indeed set $u=\psi^{2(p-1)}(x)-V(x)$ so that for $x \neq R_{i}$ we have

$$
\begin{aligned}
\Delta u & =2(p-1) \psi^{2 p-3}(\Delta \psi)+(2 p-2)(2 p-3) \psi^{2 p-4}|\nabla \psi|^{2} \\
& \geqq 2(p-1) \psi^{2 p-3}(\Delta \psi)=2(p-1) \psi^{2 p-3}\left(\psi^{2 p-1}-\varphi \psi\right) .
\end{aligned}
$$

The function $u$ achieves its maximum at some point $x_{0}\left(\neq R_{i}\right)$. At $x_{0}$ we have $(\Delta u)\left(x_{0}\right) \leqq 0$ and so $\psi^{2(p-1)}\left(x_{0}\right) \leqq \varphi\left(x_{0}\right) \leqq V\left(x_{0}\right)$. Thus $u\left(x_{0}\right) \leqq 0$, and so $u(x) \leqq 0$ everywhere.

IV. Proof that for the Minimizing $\psi, \int \psi^{2}(x) d x \geqq Z$ (when $p \geqq 4 / 3$ )

We start with the following remark:

Lemma 11. For any $\zeta \in C_{0}^{\infty}$

$$
-\int \frac{\Delta \psi}{\psi} \zeta^{2} d x \leqq \int|\nabla \zeta|^{2} d x
$$

Proof. Integrate by parts and use the Cauchy-Schwarz inequality.

We now prove the main estimate.

Lemma 12. When $p \geqq 4 / 3, \int \psi^{2}(x) d x \geqq Z$.

Proof. Let $\zeta_{0} \in C_{0}^{\infty}$ be a spherically symmetric function such that $\zeta_{0} \neq \equiv 0, \zeta_{0}(x)=0$ for $|x|<1$ and for $|x|>2$. Set $\zeta_{n}(x)=\zeta_{0}(x / n)$. By (17) we have,

$$
-\int \frac{\Delta \psi}{\psi} \zeta_{n}^{2} d x+\int \psi^{2 p-2} \zeta_{n}^{2} d x=\int \varphi \zeta_{n}^{2} d x
$$

Using Lemma 11, we find

$$
-\int \frac{\Delta \psi}{\psi} \zeta_{n}^{2} d x \leqq \int\left|\nabla \zeta_{n}\right|^{2} d x \leqq C n
$$

Next we claim that, if $p \geqq 4 / 3$, then

$$
\int \psi^{2 p-2} \zeta_{n}^{2} d x \leqq \varepsilon_{n} n^{2},
$$

where $\varepsilon_{n} \rightarrow 0$ as $n \rightarrow \infty$. Indeed we have

$$
\int \psi^{2 p-2} \zeta_{n}^{2} d x \leqq \int_{n<|x|<2 n} \psi^{2 p-2} d x
$$

If $2 p-2 \geqq 2$, we use the fact that $\psi^{2 p-2} \leqq C \psi^{2}$ in order to obtain (30). If $2 p-2 \leqq 2$, we use Hölder's inequality and we find

$$
\int_{n<|x|<2 n} \psi^{2 p-2} d x \leqq C\left[\int_{n<|x|<2 n} \psi^{2} d x\right]^{p-1}\left(n^{3}\right)^{2-p} .
$$

Assuming $p \geqq 4 / 3$, we deduce (30). On the other hand, since $\zeta_{n}$ is spherically symmetric, we have

$$
\int \varphi \zeta_{n}^{2} d x=\int[\varphi] \zeta_{n}^{2} d x
$$


where $[\varphi]$ denotes the spherical average of $\varphi$, i.e.,

$$
[\varphi](x)=\frac{1}{4 \pi|x|^{2}} \int_{|\Omega|=|x|} \varphi(\Omega) d \Omega=\frac{1}{4 \pi} \int_{|\Omega|=1} \varphi(|x| \Omega) d \Omega .
$$

By a result of $[1]$ (Eq. (35)) we know that

$$
[\varphi](x) \geqq\left(Z-\lambda_{0}\right) /|x|, \text { for }|x|>\operatorname{Max}_{1 \leqq i \leqq k}\left|R_{i}\right|,
$$

where $\lambda_{0}=\int \psi^{2}(x) d x$. Hence, for large $n$, we find

$$
\int \varphi \zeta_{n}^{2} d x \geqq\left(Z-\lambda_{0}\right) \int \frac{1}{|x|} \zeta_{n}^{2}(x) d x=\alpha\left(Z-\lambda_{0}\right) n^{2},
$$

for some positive constant $\alpha$. Combining (28), (29), (30), and (32), we find

$$
\alpha\left(Z-\lambda_{0}\right) n^{2} \leqq C n+\varepsilon_{n} n^{2} .
$$

As $n \rightarrow \infty$, we conclude that $Z \leqq \lambda_{0}$.

Remark. Lemma 12 can also be proved by a direct variational calculation using (4). This is given in Theorem 4.10 of reference [4].

\section{V. $\int \psi^{2}(x) d x>Z$ when $p \geqq 5 / 3$ and $k=1$ (Atomic Case)}

We assume now that $V(x)=Z|x|^{-1}$. The main result is the following:

Lemma 13. ([4], Theorem 4.13) Assume $p \geqq 5 / 3$, then $\int \psi^{2}(x) d x>Z$. In addition $\psi(x) \leqq M e^{-2(\delta|x|)^{1 / 2}}$ for some constants $M$ and $0<2 \delta<\int \psi^{2}(x) d x-Z$.

Proof. Since the solution of the problem $\operatorname{Min}\left\{\xi_{p}(\rho) \mid \rho \in D_{p}\right\}$ is unique, it follows that $\rho_{0}$-and therefore $\psi$-is spherically symmetric. In particular $\varphi=V-B \psi^{2}$ is also spherically symmetric. On the other hand by (31) we have,

$$
\varphi(\mathrm{x})=[\varphi](x) \geqq\left(Z-\lambda_{0}\right) /|x|,
$$

for $x \neq 0$. We already know that $\int \psi^{2}(x) d x \geqq Z$; suppose by contradiction that $\int \psi^{2}(x) d x=Z$. By (33) we have $\varphi \geqq 0$ and consequently (from (17))

$$
-\Delta \psi+\psi^{2 p-1} \geqq 0 \text {, }
$$

for $x \neq 0$. We now use a comparison function. Set $\tilde{\psi}(x)=C|x|^{-3 / 2}$. An easy computation shows that

$$
-\Delta \tilde{\psi}+\tilde{\psi}^{2 p-1} \leqq 0, \text { for }|x|>1,
$$

provided $0<C \leqq C_{0}$ where $C_{0}^{2 p-2}=3 / 4$. We fix the constant $0<C \leqq C_{0}$ such that

$$
\tilde{\psi}(x) \leqq \psi(x) \text { for }|x|=1
$$

(Recall that by Lemma 10, $\psi>0$ ). It follows from (34), (35), (36) and the (usual) maximum principle that $\widetilde{\psi}(x) \leqq \psi(x)$ for $|x|>1$. Since $\widetilde{\psi} \notin L^{2}(|x|>1)$, we obtain a contradiction. Therefore, $\int \psi^{2}(x) d x>Z$; finally we argue as in the proof of 
Lemma 8 and we conclude that for some $M, \psi(x) \leqq M \exp \left(-2(\delta|x|)^{1 / 2}\right)$ where $2 \delta<\int \psi^{2}(x) d x-Z$.

Remark. If the assumptions of Lemma 13 are not satisfied, it may happen that $\int \psi^{2} d x=Z$. Consider for example the functional

$$
\xi(\rho)=c \int\left|\nabla \rho^{1 / 2}(x)\right|^{2} d x+2 / 3 \int \rho^{3 / 2}(x) d x-\int V(x) \rho(x) d x+D(\rho, \rho),
$$

where $V(x)$ is given by (3). We claim that if $c \leqq 1 / 16 \pi$, then we have $\int \psi^{2}(x) d x=Z$. Indeed set $u=\psi-2 \varphi$. Recall that $-c \Delta \psi+\psi^{2}=\varphi \psi$, and

$$
\Delta \varphi=4 \pi \psi^{2} \text { if } x \neq R_{i} .
$$

Thus,

$$
\Delta u=\frac{1}{c}\left(\psi^{2}-\varphi \psi\right)-8 \pi \psi^{2} \geqq \frac{1}{c}\left(\psi^{2}-\varphi \psi\right)-\frac{1}{2 c} \psi^{2}=\frac{\psi u}{2 c} .
$$

At a point $x_{0}$ where $u$ achieves its maximum we have $(\Delta u)\left(x_{0}\right) \leqq 0$ and so $u\left(x_{0}\right) \leqq 0$ (note that $u(x) \rightarrow 0$ as $|x| \rightarrow \infty$ ). Consequently, $u \leqq 0$ everywhere and so $\varphi \geqq 0$ everywhere. Therefore, we must have $Z \geqq \int \psi^{2}(x) d x$. Since we already know that $Z \leqq \int \psi^{2}(x) d x$, it follows that $\int \psi^{2}(x) d x=Z$.

If $c>1 / 16 \pi$, one can still prove the weaker inequality

$$
c \psi \leqq \frac{1}{8 \pi} \varphi+(c-1 / 16 \pi) V,
$$

by the same type of arguments. Using (37) we have

$$
Z \leqq \int \psi^{2} d x \leqq Z(1+8 \pi(c-1 / 16 \pi))
$$

because $\varphi+\alpha V \geqq 0($ with $\alpha>0)$ implies $\int \psi^{2}(x) d x \leqq Z(1+\alpha)$.

Note that in the one center (atomic) case $\bar{\xi}_{3 / 2}$ is scale invariant. In fact, $E_{3 / 2}=\operatorname{Min} \xi_{3 / 2}=c Z^{3}$ and $\psi(x)=Z^{2} \psi(Z x)$.

\section{Proof of Theorem 1 Concluded}

We need a final Lemma.

Lemma 14. ([4], Theorem 4.2) For every $\lambda>0$ we have $\operatorname{Inf}\left\{\xi_{p}(\rho) \mid \rho \in D_{p}\right.$ and $\left.\int \rho(x) d x=\lambda\right\}=\operatorname{Inf}\left\{\xi_{p}(\rho) \mid \rho \in D_{p}\right.$ and $\left.\int \rho(x) d x \leqq \lambda\right\}$.

Proof. Let $\rho \in D_{p}$ be such that $\int \rho(x) d x<\lambda$; the Lemma is an obvious consequence of the following claim: There exists a sequence $\rho_{n} \in D_{p}$ such that $\int \rho_{n}(x) d x=\lambda$ and $\lim \inf \xi_{p}\left(\rho_{n}\right) \leqq \xi_{p}(\rho)$. As $\rho_{n}$, we choose

$$
\rho_{n}(x)=\rho(x)+\frac{k}{n^{3}} \zeta_{n}^{2}(x)
$$

where $\zeta_{n}(x)=\zeta_{0}(x / n)\left(\zeta_{0} \in C_{0}^{\infty}\right.$ is any function $\left.\zeta_{0} \not \equiv 0\right)$ and $k=\left[\lambda-\int \rho(x) d x\right] /$ $\int \zeta_{0}^{2}(x) d x$, so that $\int \rho_{n}(x) d x=\lambda$. We now check that lim inf $\xi_{p}\left(\rho_{n}\right) \leqq \xi_{p}(\rho)$. Using the subadditivity of the function $\int\left|\nabla \rho^{1 / 2}(x)\right|^{2} d x$ and the convexity of $\rho^{p}$, we find

$$
\xi_{p}\left(\rho_{n}\right) \leqq \xi_{p}(\rho)+A_{n}+B_{n}+C_{n},
$$


where

$$
\begin{aligned}
A_{n} & =\frac{k}{n^{3}} \int\left|\nabla \zeta_{n}(x)\right|^{2} d x \\
B_{n} & =\frac{k}{n^{3}} \int \rho_{n}^{p-1} \zeta_{n}^{2} d x \\
C_{n} & =\frac{k}{n^{3}} \int(B \rho) \zeta_{n}^{2} d x+\frac{k^{2}}{2 n^{6}} \int\left(B \zeta_{n}^{2}\right) \zeta_{n}^{2} d x
\end{aligned}
$$

We shall prove that $A_{n}, B_{n}, C_{n}$ tend to zero. Indeed we have

$$
A_{n}=\frac{k}{n^{2}} \int\left|\nabla \zeta_{0}(x)\right|^{2} d x \rightarrow 0
$$

Next, by Hölder's inequality

$$
B_{n} \leqq \frac{k}{n^{3}}\left[\int \rho_{n}^{p}(x) d x\right]^{p-1 / p}\left[\int \zeta_{n}^{2 p}(x) d x\right]^{1 / p} .
$$

But,

$$
\int \rho_{n}^{p}(x) d x \leqq C \int\left[\rho^{p}(x)+\left(\left(k / n^{3}\right) \zeta_{n}^{2}(x)\right)^{p}\right] d x \leqq C
$$

and so $B_{n} \rightarrow 0$. Finally, we have

$$
C_{n} \leqq \frac{k}{n^{3}}\left[\int(B \rho) \rho d x\right]^{1 / 2}\left[\int\left(B \zeta_{n}^{2}\right) \zeta_{n}^{2} d x\right]^{1 / 2}+\frac{k^{2}}{2 n^{6}} \int\left(B \zeta_{n}^{2}\right) \zeta_{n}^{2} d x
$$

and

$$
\frac{1}{n^{6}} \int\left(B \zeta_{n}^{2}\right) \zeta_{n}^{2}=\frac{2}{n} D\left(\zeta_{0}^{2}, \zeta_{0}^{2}\right)
$$

Therefore, $C_{n} \rightarrow 0$.

Proof of Theorem 1 concluded: For every $\lambda>0$ we set $E(\lambda)=\operatorname{Inf}\left\{\xi_{p}(\rho) \mid \rho \in D_{p}\right.$ and $\left.\int \rho(x) d x \leqq \lambda\right\}$. It is clear that $E(\lambda)$ is non-increasing and that $E(\lambda)$ is convex. In addition, the same proof as in Lemma 5 shows that there is a unique $\rho_{\lambda} \in D_{p}$ such that $\int \rho_{\lambda}(x) \leqq \lambda$ and $\xi_{p}\left(\rho_{\lambda}\right)=E(\lambda)$. Set $\lambda_{c}=\int \psi^{2}(x) d x$. It is clear that for $\lambda \geqq \lambda_{c}$ the function $E(\lambda)$ is constant: $E(\lambda)=E\left(\lambda_{c}\right)$; while $E(\lambda)$ is strictly decreasing on the interval $\left[0, \lambda_{c}\right]$. It follows that for $\lambda \leqq \lambda_{c}$ we have $\int \rho_{\lambda}(x) d x=\lambda$. Consequently, if $\lambda \leqq \lambda_{c}$ there is a unique solution for Problem (I). When $\lambda>\lambda_{c}$, we deduce from Lemma 14 that Problem (I) has no solution.

By the same methods as used in Lemma 6 , the unique minimizing $\psi$ for $E(\lambda)$, $\lambda \leqq \lambda_{c}$ satisfies

$$
-\Delta \psi+U_{\psi} \psi=-\phi_{0} \psi
$$

where,

$$
U_{\psi}=\psi^{2 p-2}-V+B \psi^{2}
$$

It is also true (as shown in [1] for the TF problem) that $E(\lambda)$ is differentiable and

$$
-\phi_{0}(\lambda)=\partial E / \partial \lambda
$$


Since $\psi$ satisfies (38) and $\psi(x)>0$ (by Harnack's inequality as in Lemma 10), we can conclude that $\psi$ and $\phi_{0}$ are, in fact, the lowest eigenfunction and eigenvalue of $-\Delta+U_{\psi}(x)$.

To summarize what has been proved so far, the function $E(\lambda)$ has many features in common with the $E(\lambda)$ for TF theory [1]. It is convex, non-increasing and has an absolute minimum at some $\lambda_{c}<\infty$ beyond which $E(\lambda)$ is constant. One important difference is that $\lambda_{c}>Z$, at least for the atomic case and $p \geqq 5 / 3$. There is another important difference: in TF theory, $E(\lambda) \sim-c \lambda^{1 / 3}$ for small $\lambda$, i.e., $\partial E /\left.\partial \lambda\right|_{\lambda=0}=-\infty$. In TFW theory, this is not so as the following shows.

Lemma 15. Let $e_{0}$ be the lowest eigenvalue (ground state energy) of the Schrödinger operator $-\Delta-V(x)$ with $V$ given by (3). Then

$$
\begin{gathered}
\lim _{\lambda \downarrow 0} \lambda^{-1} E(\lambda) / e_{0}=1, \text { i.e., } \\
-\phi_{0}(0)=\partial E(\lambda) /\left.\partial \lambda\right|_{\lambda=0}=e_{0} .
\end{gathered}
$$

Proof. Let $\varphi$ be the normalized eigenfunction of $-\Delta-V(x)$ belonging to $e_{0}$ and let $\rho_{\lambda}(x)=\lambda \varphi(x)^{2}$. Then $\xi_{p}\left(\rho_{\lambda}\right) \leqq \lambda e_{0}+O(\lambda)$ since the terms in $\xi_{p}$ of degree higher than the first, while they are positive, are finite and $0(\lambda)$. Conversely,

$$
E(\lambda) \geqq \inf _{\rho}\left\{\int\left|\nabla \rho^{1 / 2}\right|^{2}-\int V \rho \mid \int \rho=\lambda\right\},
$$

but this is precisely the variational problem for the Schrödinger Equation.

Yet another important distinction with TF theory should be noted. $\psi(x)$ is never zero and therefore $\rho(x)=\psi(x)^{2}$ does not have compact support. In TF theory, $\rho$ has compact support [1] whenever $\lambda<\lambda_{c}$.

\section{Binding of Atoms in TFW Theory}

Binding does not occur in TF theory [1]; that is Teller's Theorem. Binding can occur in TFW theory as we shall now prove in a special case.

First it is necessary to have a clear definition of what binding means. Given the nuclear coordinates $R_{1}, \ldots, R_{k}$, define

$$
\tilde{E}\left(\lambda ;\left\{R_{i}\right\}\right) \equiv E\left(\lambda ;\left\{R_{i}\right\}\right)+U\left(\left\{R_{i}\right\}\right)
$$

where

$$
U\left(\left\{R_{i}\right\}\right)=\sum_{1 \leqq i<j \leqq k} z_{i} z_{j}\left|R_{i}-R_{j}\right|^{-1} .
$$

Let us start with two neutral atoms infinitely far apart. The total energy is then $A_{12}=E_{1}\left(z_{1}\right)+E_{2}\left(z_{2}\right)$ where $E_{i}$ is the energy of an atom with nuclear charge $z_{i}$. The next step is to distribute the total electron charge so as to minimize the total energy, namely

$$
B_{12}=\min _{0 \leqq \lambda \leqq z_{1}+z_{2}}\left(E_{1}\left(z_{1}+z_{2}-\lambda\right)+E_{2}(\lambda)\right) .
$$

It may be, and usually is the case in TFW theory, that $B_{12}<A_{12}$, i.e., ions are more stable than atoms. Finally, we bring the atoms together and define

$$
C_{12}=\inf _{R_{1}, R_{2}} \tilde{E}\left(z_{1}+z_{2} ; R_{1}, R_{2}\right) .
$$

If $C_{12}<B_{12}$, then binding occurs. 
To show that binding is possible, suppose that $z_{2}$ is sufficiently small compared to $z_{1}$ so that the following is satisfied

(a)

(b)

$$
\left(\partial E_{1} / \partial \lambda\right)\left(\frac{z_{1}+\lambda_{c}^{1}}{2}\right)<-z_{2}^{2} / 4,
$$

$$
\lambda_{c}^{1}-z_{1} \geqq 2 z_{2} \text {, }
$$

where $\lambda_{c}^{1}$ is the critical $\lambda$ for atom 1 ; then we claim that

$$
B_{12}=E_{1}\left(z_{1}+z_{2}\right) \text {. }
$$

This follows from the following observation: $\left(\partial E_{2} / \partial \lambda\right) \geqq\left(\partial E_{2} / \partial \lambda\right)(0)=-z_{2}^{2} / 4$ by Lemma 15 and convexity. Then the equation $\left(\partial E_{1} / \partial \lambda\right)\left(z_{1}+z_{2}-\lambda\right)=$ $\left(\partial E_{2} / \partial \lambda\right)(\lambda)$ cannot have a solution for $0 \leqq \lambda \leqq z_{1}+z_{2}$.

For two such atoms the lowest total energy occurs when the smaller atom is completely stripped of its electrons which become attached to the larger atom. Now consider the first atom with $R_{1}=0$ and with $\lambda=z_{1}+z_{2} \leqq \lambda_{c}^{1}$. The electric potential $\varphi$ which is spherically symmetric, will be negatıve for large $R$. If the second nucleus $z_{2}$ is placed at a point $r$ where $\varphi(r)<0$, the total energy will be reduced by an amount $z_{2} \varphi(r)$. Thus,

$$
C_{12}<B_{12}+z_{2} \varphi(r)
$$

and binding occurs.

Acknowledgements. One of the authors (H.B.) would like to thank the following for their hospitality during the course of this work: The Princeton University, Courant Institute and The University of Chicago.

\section{References}

1. Lieb, E. H., Simon, B. : Adv. Math. 23, 22-116 (1977)

2. von Weizsäcker, C. F. : Z. Phys. 96, 431-458 (1935)

3. Kompaneets, A. S., Pavloskii, E. S. : Sov. Phys. JETP 4, 328-336 (1957)

4. Benguria, R. :The von Weizsäcker and exchange corrections in the Thomas-Fermi theory.Princeton University Thesis: June 1979 (unpublished)

5. Balàzs, N. L. : Phys. Rev. 156, 42-47 (1967)

6. Gombás, P. : Acta Phys. Hung. 9, 461-469 (1959)

7. Stampacchia, G. : Equations elliptiques du second ordre à coefficients discontinus. Montreal: Presses de l'Univ. 1965

8. Bers, L., Schechter, M. : Elliptic equations in Partial Differential Equations. New York : Interscience pp. 131-299. 1964

9. Trudinger, N. : Ann. Scuola Norm. Sup. Pisa 27, 265-308 (1973)

Communicated by A. Jaffe

Received June 9, 1980 